\title{
IN-USE STABILITY STUDIES OF TWO VETERINARY MEDICINAL PRODUCTS: ALBENDAZOLE AND OXYTETRACYCLINE
}

\author{
Y.M.M. YAGOUB *; SIHAM ABDOUN ${ }^{* *}$ and H.I. SERI ${ }^{* * *}$ \\ *Veterinary Medicine Department, Gingko Investment Co, LTD-Sudan. \\ ***National Drug Quality Control Laboratory; National Medicine and Poisons Board, Sudan. \\ ${ }^{* * *}$ Department of Animal Health and Surgery, College of Veterinary Medicine, Sudan University of Science and Technology. \\ Corresponding author: Hisham Ismail Seri; College of Veterinary Medicine; Sudan University of Science and Technology. \\ P.O. Box: 204 Khartoum North, Sudan. E-mail: hishamseri@Yahoo.com
}

\section{ABSTRACT}

Received at: 2/12/2012

Accepted: 5/1/2013
The in-use stability of Albendazole and oxytetracycline, Veterinary medicinal products commonly used in animal health and production, was investigated in suspension formulation (albendazole) or injectable formulation (Oxytetracycline), according to stability guidelines (ICH, 2003). The degradation process, if any, was monitored by an HPLC method. The change in colour using visual inspection and $\mathrm{pH}$ value using $\mathrm{pH}$ meter, was also monitored for 7 days (Oxytetracycline) and 2 months (Albendazole). Albendazole and Oxytetracycline, showed few to prominent degradation in active ingredient concentration and $\mathrm{pH}$ value in solution when exposed to inuse environment mimicking the storage conditions recommended by the manufacturer. The obtained results are of interest for stability studies and/or quality control purposes of Albendazole and Oxytetracycline commercial products.

Keywords: In use stability, Albendazole, Oxytetracycline

\section{INTRODUCTION}

In-use stability testing aims to provide information for the labelling on the preparation, storage condition and utilization period of multi dose products after opening, reconstitution or dilution of a solution, e.g. an antibiotic injection supplied as powder for reconstitution. As far as possible the test should be designed to simulate the use of the finished pharmaceutical product in practice, taking into consideration the filling volume of the container and any dilution or reconstitution before use. At interval comparable to those which occur in practice appropriate quantities should be removed by the withdrawal methods normally used and described in the product literature (WHO, 2009).

In Sudan, as in other tropical and subtropical countries, worm infestations continue to represent a serious health hazard. The most common drugs used as anthelmintics belong to the benzimidazole series. Albendazole, Fenbendazole (FEN) and Mebendazole (MEB) (Cook, 1990; Wen et al., 1993, and Gomes and Nagaraju, 2001), represents one of the most commercialized benzimdazole in the world.

Due to their chemical features, all the benzimidazole drugs are sensitive to light, with behaviour common to all members of this class of compounds (Del Brutto et al., 1993; Al-Kurdi et al., 1999). The amine derivative from hydrolysis of the carbamic group has been reported to be the main photo-degradation product, indicated also by metabolism studies as the major metabolite of the drugs (Del Brutto et al., 1993). Accordingly, a close monitoring of these drugs, as such or in pharmaceutical formulations, constitutes a noteworthy analytical problem in regions presenting tropical climate.

Albendazole oral suspension is an aqueous vehicle. It contains one or more preservatives and dispersing or suspending agents. It contains not less than 90.0 percent and not more than 110.0 percent of the labeled amount of $\mathrm{C}_{12} \mathrm{H}_{15} \mathrm{~N}_{3} \mathrm{O}_{2} \mathrm{~S}$. The $\mathrm{pH}$ of the final product was set between 4.5 and 5.5 (USP, 2011).

Antibiotics are used in veterinary medicine due to their therapeutic value and in animal production as additives to enhance growth and food efficiency. Tetracycline antibiotics are widely used in the prevention and treatment of infectious diseases and as food additives for animal growth promotion. Many tetracyclines are available commercially and permitted for human administration, such as oxytetracycline, chlortetracycline, and doxycycline.

Oxytetracycline for injection is sterile material consisting of oxytetracycline hydrochloride with or without excipients. It is supplied in a sealed container. It contains $90.0-120.0 \%$ of oxytetracycline hydrochloride: $\left(\mathrm{C}_{22} \mathrm{H}_{24} \mathrm{~N}_{2} \mathrm{O}_{9} \mathrm{HCL}\right)$ of the prescribed or stated amount. $\mathrm{pH}$ of the $10 \% \mathrm{w} / \mathrm{v}$ solution is $2.0-3.0$. 
The objective of this study was to determine the in-use stability parameters of two commercially available veterinary products each from albendazole and oxytetracycline, stored under ideal conditions, in Khartoum North Sudan.

\section{MATERIALS and METHODS}

Site of study: For the purpose of this study, a store that belongs to Gingko pharmaceutical company was used. The location was: House No.178, Block No.11, Kafouri, Khartoum North.

Storage condition: Standard conditions according to the rules of Good Storage Practice (GSP) approved by World Health Organization guidlines. The store was equipped with cooling and ventilation facility, that allow to maintain stable temperature $\left(25^{\circ} \mathrm{C}\right)$ and humidity (40) conditions.

Experimental drugs: In this study, the following veterinary drugs were used:

1. Oxytetracycline $\mathrm{HCl} 5 \%-100 \mathrm{ml}$ injection (Oxyko ${ }^{\circledR} 5 \%$, Gingko- China).

2. Oxytetracycline $\mathrm{HCl} 5 \%-100 \mathrm{ml}$ injection (Oxtra ${ }^{\circledR}$ Italy).

3. Albendazole oral suspension 2.5\%-1000 ml (Albenko $^{\circledR}$, Gingko-China).

4. Albendazole oral suspension 2.5\%-1000 ml (Albendazole, pharma swede medicine, Egypt).

From each of the above mentioned drugs we did choose two different batches and from each batch one vial was selected randomly (the total was 8 vials).

Working standards and reagents: The following reagents were obtained as follows:

Albendazole working standard powder $99.07 \%$, (Changzhou yabang- qg pharmachem co, ltd, China). Oxytetracycline $\mathrm{HCl}$ working standard powder $95.5 \%$, (Shandong jinyang pharmaceutical Co, ltd, China). Tetrabutylammonium hydrogen sulphate for HPLC 99.9\%, (Lopachemie, India). Disodium edetate powder for HPLC 99\%, (Techno pharmacheme. India). Monobasic sodium phosphate, for HPLC, 99\%, (Techno pharmacheme. India). Dibasic potassium phosphate, for HPLC $99 \%$, (Ttechno pharmacheme. India). Tertiary butyl alcohol, for HPLC $99.1 \%$ (Lobachemie, India). Methanol for HPLC 99.8\%, (Lobachemie, India). HCL 35\% - 38\%, (Lobachemie, India). Buffer solution $\mathrm{pH} 4.00$, (Scharlab, Spain). Buffer solution pH 10.01 (HANA. Roman).

In-use stability study: Here, we tested the stability of colour, $\mathrm{pH}$, and pharmacopeia assay; the drugs were kept in ideal storage conditions as described above.

Oxytetracycline $\mathrm{HCl}$ : daily $5 \mathrm{ml}$ aliquots were withdrawn from each vial for seven continuous days. Samples for testing were collected directly from each vial at the first day of experiment and after 7 days following opening of the vials.

Albendazole: weekly $5 \mathrm{ml}$ aliquots were withdrawn from each vial and drooped off for four consecutive weeks. Samples for testing were collected directly from each container at the first day of experiment and then after 30 and 60 days, following the opening of the container.

Parameters tested: The following parameters were tested in every drug as scheduled:

$p H$ : The $\mathrm{pH}$ of each drug was measured using $\mathrm{pH}$ meter (HANA 211, Romaine).

The colour: The evaluation of the colour by visual test according to methods used in international pharmacopoeia is not practical because the reagents are not available, so we used digital camera and make photos for each sample at the predetermined time points, after that we compared between the photos using computer colour techniques.

Assay: An HPLC method (USP, 2011) for oxytetracycline injection and (USP, 2011) HPLC method for Albendazole were used to evaluate the stability of the active ingredient in the experimental drugs. For this purpose a CECILL 110 series, England, connected with UV detection (210-380nm) high performance variable wave length monitor, CICEILL 1200, England, with pump, injector, column, computer, injection and degaser device, all from CICEILL company, was used.

\section{RESULTS}

Albendazole oral suspension results: The colour of the Albendazole suspension in the two products tested did not show any change during the storage period (60 days). The first product was a yellow or straw yellow suspension and the second one was almost white to yellowish suspension and continued to be like that for the three time points of the test.

The $\mathrm{pH}$ values of the four tested samples as shown in table (1) showed minor to prominent changes in their values with time but they are still within the recommended values. The assay concentration $\%$ of the four tested samples as shown in table (2) also exhibited prominent deterioration below the recommended level.

Oxytetracycline 5\% injectable solution: There is no change in colour of oxytetracycline (An amber clear liquid) samples tested. The $\mathrm{pH}$ values of the four tested samples as shown in table (3) were within the same value of time zero with no change during the 7 days of the study. The four tested compounds showed minor to moderate changes in the active ingredient concentration at day seven following withdrawal of the first sample as shown in table (4). 
$\underline{\text { Assiut Vet. Med. J. Vol. } 59 \text { No. } 136 \text { January } 2013}$

Table 1: Comparison of the $\mathrm{pH}$ results of albendazole suspension

\begin{tabular}{cccc}
\hline Sample & Day zero & Day thirty & Day sixty \\
\hline Albendazole 1 & 4.87 & 4.70 & 4.65 \\
\hline Albendazole 2 & 4.90 & 4.77 & 4.68 \\
\hline Albendazole 3 & 4.81 & 4.66 & 4.67 \\
\hline Albendazole 4 & 5.00 & 4.85 & 4.83 \\
\hline
\end{tabular}

Table 2: Comparison of active ingredient concentration (\%content) of albendazole suspension

\begin{tabular}{cccc}
\hline Sample & Day zero time & Day thirty & Day sixty \\
\hline Albendazole 1 & 94.0 & 88.1 & 89.1 \\
\hline Albendazole 2 & 95.6 & 83.5 & 83.4 \\
\hline Albendazole 3 & 95.6 & 88.2 & 79.5 \\
\hline Albendazole 4 & 95.6 & 85.3 & 74.6 \\
\hline
\end{tabular}

Table 3: Comparison of $\mathrm{pH}$ test results of Oxytetracycline injection

\begin{tabular}{ccc}
\hline Sample & Day zero & Day 7 \\
\hline Oxytetracycline 1 & 8.5 & 8.5 \\
\hline Oxytetracycline 2 & 8.2 & 8.2 \\
\hline Oxytetracycline 3 & 8.6 & 8.6 \\
\hline Oxytetracycline 4 & 8.4 & 8.4 \\
\hline
\end{tabular}

Table 4: Comparison of active ingredient of Oxytetracycline injection

\begin{tabular}{ccc}
\hline Sample & Day Zero (\% content) & Day 7(\% content) \\
\hline Oxytetracycline 1 & 99.0 & 94.5 \\
\hline Oxytetracycline 2 & 99.9 & 94.9 \\
\hline Oxytetracycline 3 & 100.5 & 96.1 \\
\hline Oxytetracycline 4 & 96.2 & 96.0 \\
\hline
\end{tabular}

\section{DISCUSSION}

In the present paper, the degradation study was performed using controlled storage conditions, provided with humidity and a temperature controller. The compounds were monitored by HPLC and also changes in colour and $\mathrm{pH}$ were characterized.

The $\mathrm{pH}$ values of the four samples of albendazole were within the recommended by USP (4.5-5.5). There is prominent change in $\mathrm{pH}$ values within the two months duration of the experiment, although they were again within the recommended range, but this may indicate further change may occur under filed and/or improper storage conditions.

Following storage for one and two months respectively, albendazole expressed noticeable change in assay $\%$ down to $74.6 \%$. Albendazole, Mebendazole and Fenbendazole showed high photosensitivity in 
solution but a reliable stability in solid form and when exposed to a temperature up to $50{ }^{\circ} \mathrm{C}$. The thermal degradation test, performed under temperatures from 20 to $50{ }^{\circ} \mathrm{C}$, demonstrated a very high stability both in solid and solution form. These temperature values were considered a reliable range that can be reached in the drug storages of the tropical lands. These results confirmed that the degradation process was caused just by light and not by high temperature (Ragno et al., 2006). So here we may attribute the degradation to the change in $\mathrm{pH}$ values, since the temperature, humidity, and light were controlled in the store.

As witnessed here, there was no change in oxytetracycline colour in the two investigated products. This may be attributed either to the short duration of the study as well as to the ideal storage conditions during the study period. The sealed containers should be protected from light as recommended by British Pharmacopeia (BP, 2012). Oxytetracycline is stable in air but exposure to strong sun light causes it to darken. It deteriorates in solution with $\mathrm{pH}$ below 2 and is rapidly destroyed by alkali hydroxide (Osol and Hoover, 1975).

There were no change in $\mathrm{pH}$ values of the four oxytetracycline tested samples and the values were within that recommended by the manufacturer. The use of inert gases and antioxidants was reported to be essential for retarding the rapid oxidative decomposition of OTC solutions (Chas, 1955). Propylene glycol; pyridine derivatives; and pantothenic, lactic, and tartaric acids may be used as stabilizing agents in addition to storage of tetracycline solution at a cool temperature (Ali, 1984). Weinstein et al. (1959) reported that oxytetracycline (OTC) intramuscular solution in $67 \%$ propylene glycol was stable for 2 years at room temperature. Without the use of propylene glycol, the product was stable only for 2 days. A $200 \mathrm{mg} / \mathrm{ml}$ solution of OTC in $40 \% \mathrm{w} / \mathrm{v} 2$ pyrrolidone was found to have excellent physical and chemical stability over a wide range of storage temperatures (Aguiar et al., 1987).

The four tested compounds showed minor to moderate changes in the active ingredient concentration (assay $\%$ ) at day seven following first sample opening. Under abnormal conditions (heat, $\mathrm{pH}$, and humidity), tetracyclines undergo reversible epimerization at positions C-4 and C- 6 to form a mixture of degradation products. These degradation products or contaminants have very low antibiotic activity; in addition, some of them can be toxic (Mitscher, 1978).

Tetracyclines exhibit general poor stability and during storage in animal feeds and premixes may be subjected to extensive degradation, such as epimerization (Liang et al., 1998).

Tetracycline antibiotics are known to undergo rapid decomposition in aqueous solutions, which was reported to be pH-dependent (Stephens et al., 1956; Doerschuk et al., 1955; McCormick et al., 1956; McCormick et al., 1957; Hussar et al., 1968).

The degradation of tetracyclines is complex and the drugs are subject to decomposition in both acidic and alkaline media (Izer et al., 1994). Vej- Hansen et al. (1978) studied the degradation of oxytetracycline (OTC) in aqueous solutions over the $\mathrm{pH}$ range of $0.5-$ 12 at $60^{\circ} \mathrm{C}$ and an ionic strength of $0.5 \mathrm{M}$. They found that OTC decomposition followed first-order kinetics at any given $\mathrm{pH}$ and from $\mathrm{pH} 3.5$ to 10 ; the degradation rate was practically independent of $\mathrm{pH}$. In contrast, kinetic studies carried out by Cornwell (1980) with OTC revealed that from $\mathrm{pH} 2.0$ to 6.0, first-order kinetics were not found, but rather an initial equilibration occurred which was followed by a much slower rate of degradation.

It is to be concluded that, monitoring of veterinary products for in-use stability is of value in attaining therapeutic goals. Both products tested in this study showed minor to moderate degradation in some of the stability parameters tested. This could be of value in quality control purposes of Albendazole and Oxytetracycline commercial products.

\section{REFERENCES}

Aguiar, A.J.; Armstrong, W.W. and Desai, S.J. (1987): Development of oxytetracycline long-acting injectable, J. Controlled Release, 6: 375-385.

Ali, S.L. (1984): Tetracycline hydrochloride, in Analytical Profile of Drug Substances (K. Flory, ed.), Academic Press, Inc., New York, Vol. 13, pp. 597-653.

Al-Kurdi, Z.; Al-Jallad, T.; Badwan, A. and Jaber, A. M.Y. (1999): High Performance Liquid Chromatography Method For Determination Of Methyl-5-Benzoyl-2-Benzimidazole Carbamate (Mebendazole) And Its Main Degradation Product In Pharmaceutical Dosage Forms. Talanta, 50: 1089-1097.

B.P. (2012): British Pharmacopoeia (Veterinary) specific monograph of oxytetracyciline injection.

Chas (1955): Pfizer and Co., Inc., Brit. Patent, 742, 156.

Cook, G.C. (1990): Use of benzimidazole chemotherapy in human helminthiases: indications and efficacy. Parasitol Today. 6(4): 133-136.

Cornwell, R.L. (1980): Evaluation of a long-acting injectable oxytetracycline, Mod. Vet. Pract., 61: 945-947.

Del Brutto, O.H.; Sotelo, J. and Roman, G.C. (1993): Therapy for neurocysticercosis: a reappraisal. Clin. Infect. Dis. 17:730-735. 
Doerschuk, A.P.; Bitler, B.A. and McCormick, J.R.D. (1955): Reversible isomerizations in the tetracycline family, J. Am. Chem. Soc., 77, 4687.

Gomes, A.R. and Nagaraju, V. (2001): Highperformance liquid chromatographic separation and determination of the process related impurities of mebendazole, fenbendazole and albendazole in bulk drugs. J. Pharm. Biom. Anal., 26: 919-927.

Hussar, D.A.; Niebergall, P.J.; Sugita, E.T. and Doluisio, J.T. (1968): Aspects of the epimerization of certain tetracycline derivatives, J. Pharm. Pharmacol., 20 (7): 539-546.

ICH "International Conference on Harmonization" (2003): guideline Q1A (R2). Stability testing of new drug substances and products. Federal Register, 3, 29103.

Izer, K.; Torok, I. and Pinter-Magyar, P. (1994): Stability of oxytetracycline hydrochloride in eye-drops, prepared in pharmacies, Acta Pharm. Hung., 64: 63-66.

Liang, Y.M.; Denton, B. and Bates, R.B. (1998): Stability studies of tetracycline in methanol solution. J. Chromatogr. A 827: 45-55 (1998).

McCormick, J.R.D.; Fox, S.M.; Smith, L.L.; Bitler, B.A.; Reichenthal, J.; Origoni, V.E.; Muller, W.H.; Winterbottom, $R$. and Doerschuk, A.P. (1956): On the nature of the reversible isomerization occurring in the tetracycline family, J. Am. Chem. Soc., 78: 3547-3548.

McCormick, J.R.D.; Fox, S.M.; Smith, L.L.; Bitler, B.A.; Reichenthal, J.; Origoni, V.E.; Muller, W.H.; Winterbottom, R. and Doerschuk, A.P. (1957): Studies of the reversible epimerization occurring in the tetracycline family. The preparation, properties and proof of structure of some 4- epi-tetracyclines, J. Am. Chem. Soc., 79: 2849-2858.

Mitscher, L.A. (1978): The Chemistry of the Tetracycline Antibiotics; Medical Research Series, vol. 9. Marcel Dekker, New York, NY, p. 123.

Osol, A. and Hoover (eds.), J.E. (1975): Remington's Pharmaceutical Sciences. $15^{\text {th }}$ ed. Easton, Pennsylvania: Mack Publishing Co., p. 1142.

Ragno, G.; Risoli, A.; Ioele, G. and De Luca, M. (2006): Photo- and Thermal-Stability Studies on Benzimidazole Anthelmintics by HPLC and GC-MS. Chem. Pharm. Bull. 54(6): 802-806.

Stephens, C.R.; Conover, L.H.; Gordon, P.N.; Pennington, F.C.; Wagner, R.L.; Brunings, K.J. and Pilgrim, F.J. (1956): Epitetracycline-The chemical relationship between tetracycline and "Quatrimycin', J. Am. Chem. Soc., 78: 1515-1516.

USP (2011): United States Pharmacopoeia USP 34, NF 29, page 1191, 1771.

Vej-Hansen, B.; Bungaard, H. and Kreilgard, B. (1978): Kinetics of degradation of oxytetracycline in aqueous solution, Arch. Pharm. Chem. Sci. Ed., 6: 151-163.

Weinstein, H.; Lidsky, J.S. and Delahunt, C.S. (1959): Preconstituted oxytetracycline intramuscular solution, a new preparation, Antibiot. Med. Clin. Ther., 6: 526-533.

Wen, H.; New, R.R.C. and Craig, P.S. (1993): Diagnosis and treatment of human Hydatidosis. Br. J.Clin.Pharm. 35: 565- 574.

WHO (2009): Annex 2 Stability testing of active pharmaceutical ingredients and finished pharmaceutical products. Technical Report Series, No. 953, 2009.

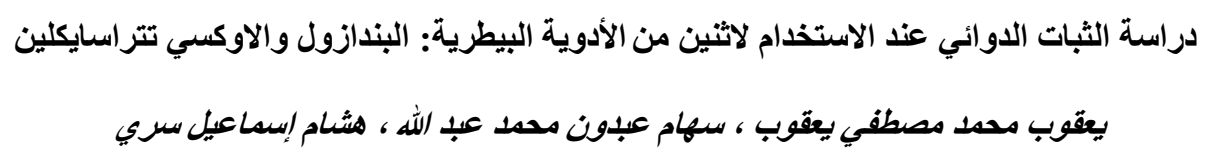

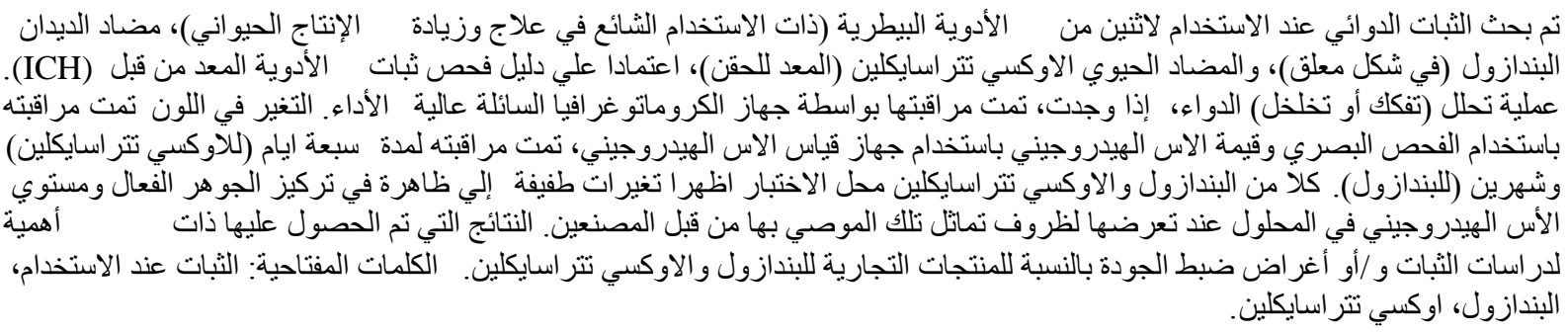

